\title{
Composição taxonômica da assembleia de larvas de peixes no reservatório de Rosana, Rio Paranapanema, Brasil
}

\author{
Darlon Kipper ${ }^{1,2}$, Andréa Bialetzki ${ }^{1,2,3}$ \& Miriam Santin ${ }^{1,2}$ \\ ${ }^{1}$ Laboratório de Ictioplâncton, Núcleo de Pesquisas em Limnologia, Ictiologia e Aquicultura, \\ Universidade Estadual de Maringá - UEM, Av. Colombo, n. 5790, bloco G-80, sala 12, \\ CEP 87020-900, Maringá, PR, Brasil \\ ${ }^{2}$ Pós-graduação em Ecologia de Ambientes Aquáticos Continentais, Departamento de Biologia, \\ Universidade Estadual de Maringá - UEM \\ ${ }^{3}$ Autor para correspondência: Andréa Bialetzki,e-mail: bialetzki@nupelia.uem.br
}

KIPPER, D., BIALETZKI, A. \& SANTIN, M. Taxonomic composition of the assemblage of fish larvae in the Rosana reservoir, Paranapanema River, Brazil. Biota Neotrop. 11(1): http://www.biotaneotropica.org.br/ v11n1/en/abstract?short-communication+bn00911012011.

\begin{abstract}
There are few studies in reservoirs that consider the Early Life Stages (ELS) of fish. Information on the ecology of fish larvae are useful for understanding the biology of the species, since ELS represent a critical period in fish life cycle. In this context, knowledge about the assemblages of larvae can be valuable for the prediction and understanding of recruitment, thus assisting in the management of the environment. Therefore, this study intends to investigate the composition of the assemblage of fish larvae in different regions of Rosana reservoir. Samples were collected quarterly (March, June, September and December) in 2002, in the limnetic and littoral regions of the zones fluvial, transition and lacustrine of reservoir. In the laboratory, the material was sorted and larvae were separated and identified to lowest possible taxonomic level. The taxa that were the major contributors in the limnetic region were Plagioscion squamosissimus (Perciformes), Hypophthalmus edentatus, Pimelodus maculatus (Siluriformes), Astyanax spp. and Roeboides descalvadensis (Characiformes), whereas in the coastal region Bryconamericus stramineus and Apareiodon affinis (Characiformes) prevailed. Limnetic and littoral regions of the reservoir, as well as their respective zones showed differences in larvae species composition and segregation of assemblages can be influenced by a preference for habitats or evolutionary questions involving reproductive strategies of species.
\end{abstract}

Keywords: ichthyoplankton, limnetic region, littoral region, reservoir.

KIPPER, D., BIALETZKI, A. \& SANTIN, M. Composição taxonômica da assembleia de larvas de peixes no reservatório de Rosana, Rio Paranapanema, Brasil. Biota Neotrop. 11(1): http://www.biotaneotropica.org.br/ v11n1/pt/abstract?short-communication+bn00911012011.

Resumo: Em reservatórios, são escassos os estudos que consideram as fases iniciais do ciclo de vida de peixes, sendo que informações sobre a ecologia de larvas de peixes são úteis para o entendimento da biologia das espécies, visto que os estágios iniciais representam um período crítico no ciclo de vida dos peixes. Neste contexto, o conhecimento a respeito das assembleias de larvas pode ser valioso para a predição e o entendimento do recrutamento, auxiliando desta maneira, no manejo do ambiente. Desta forma, o presente trabalho pretende verificar a composição da assembleia de larvas de peixes em diferentes regiões do reservatório. As coletas foram realizadas trimestralmente (março, junho, setembro e dezembro) no ano de 2002, nas regiões limnética e litorânea das zonas fluvial, transição e lacustre do reservatório de Rosana. No laboratório, o material foi triado e as larvas foram separadas e identificadas ao menor nível taxonômico possível. Os táxons que tiveram maior contribuição na região limnética foram Plagioscion squamosissimus (Perciformes), Hypophthalmus edentatus, Pimelodus maculatus (Siluriformes), Astyanax spp. e Roeboides descalvadensis (Characiformes), enquanto na região litorânea Bryconamericus stramineus e Apareiodon affinis (Characiformes). As regiões limnética e litorânea do reservatório, assim como suas respectivas zonas apresentaram diferenças na composição de espécies de larvas e a segregação das assembleias pode estar sendo influenciada pela preferência por habitats ou por questões evolutivas que envolvem as estratégias reprodutivas das espécies.

Palavras-chave: ictioplâncton, região limnética, região litorânea, reservatório. 


\section{Introdução}

Nos reservatórios, é possível visualizar a formação de compartimentos de grande complexidade espacial e temporal, devido às próprias características do relevo inundado, padrões de circulação/retenção e interações com os tributários e o ambiente terrestre (Straškraba et al. 1993). Thornton et al. (1990) propuseram o zoneamento dos reservatórios, onde ao longo do eixo longitudinal há um contínuo, com início na região de influxo do rio até a barragem. Segundo estes autores, neste contínuo, três zonas distintas são observadas: fluvial (incluindo a região do delta), transição e lacustre. Além destas, também podem ser distinguidas duas regiões transversais, a limnética e a litorânea (Esteves 1998). Todas se diferenciam quanto às propriedades físicas, químicas e biológicas, causando heterogeneidade espacial e temporal e, consequentemente, promovendo a diversidade de habitats, que é um fator decisivo para a organização das comunidades e fundamental para a diversidade biológica (Tundisi 1999).

Na literatura, há uma ampla discussão acerca da importância relativa dos fatores físicos e biológicos na definição de padrões de distribuição espacial de peixes em ambientes lênticos (Oliveira \& Goulart 2000), tais como os reservatórios. Segundo estes autores, há um conjunto de fatores responsáveis pela determinação dos padrões de distribuição espacial dos peixes nos diferentes ambientes lênticos, e a importância relativa de cada fator varia amplamente entre as assembleias de cada local.

Os estágios iniciais de desenvolvimento dos peixes, assim como os adultos, apresentam diferentes padrões de distribuição, tanto espaciais quanto temporais. A estrutura da assembleia de larvas de peixes é diretamente influenciada pelo modo, local, período, duração e intensidade reprodutiva (Bialetzki et al. 2005). Prover informações a respeito do recrutamento, talvez seja o fator primordial que motiva os estudos das assembleias de ictioplâncton (Miller 2002). O conhecimento das forças que determinam a forma de organização destas assembleias ao longo do espaço e do tempo pode ser valioso para a predição e o entendimento do recrutamento, auxiliando desta maneira, no manejo do ambiente.

Neste contexto, a proposta deste trabalho foi conhecer a composição da assembleia de larvas de peixes no reservatório de Rosana, rio Paranapanema. Especificamente, pretende-se investigar padrões de composição das larvas das espécies presentes em distintas unidades de hábitat (regiões limnética e litorânea e suas respectivas zonas: fluvial, de transição e lacustre).

\section{Material e Métodos}

O reservatório de Rosana (Figura 1), inserido na bacia do Rio Paranapanema, foi fechado em novembro de 1986 e possui uma área inundada de $220 \mathrm{~km}^{2}$. O eixo da barragem se localiza entre os municípios de Diamante do Norte (PR) e Primavera (SP), a 20 km de sua foz, no Rio Paraná. Há duas unidades de conservação em suas margens: o Parque Estadual do Morro do Diabo, à direita, e a Estação Ecológica do Caiuá, à esquerda. Apesar disso, a maior parte de seu entorno está ocupada por pastagens. Macrófitas submersas, especialmente Egeria densa, têm grande abundância em termos de distribuição espacial e cobertura (profundidades inferiores a $5 \mathrm{~m}$ ) (Júlio Júnior et al. 2005).

As coletas foram realizadas trimestralmente (março, junho, setembro e dezembro) no ano de 2002, nas zonas fluvial, de transição e lacustre do reservatório. Em cada zona foram escolhidos dois pontos de coleta, sendo um na região limnética e outro na litorânea. Devido ao fato dos organismos do ictioplâncton diferirem em tamanho, distribuição horizontal, comportamento, disponibilidade temporal e susceptibilidade aos vários aparelhos de captura, foram utilizadas dois tipos de amostragens: i) Na região limnética - redes de plâncton do tipo cônico-cilíndrica, malha $0,5 \mathrm{~mm}$, área da boca da rede de $0,1104 \mathrm{~m}^{2}$, as quais foram arrastadas na superfície da água, por 10 minutos, sendo estas equipadas com fluxômetro para a obtenção

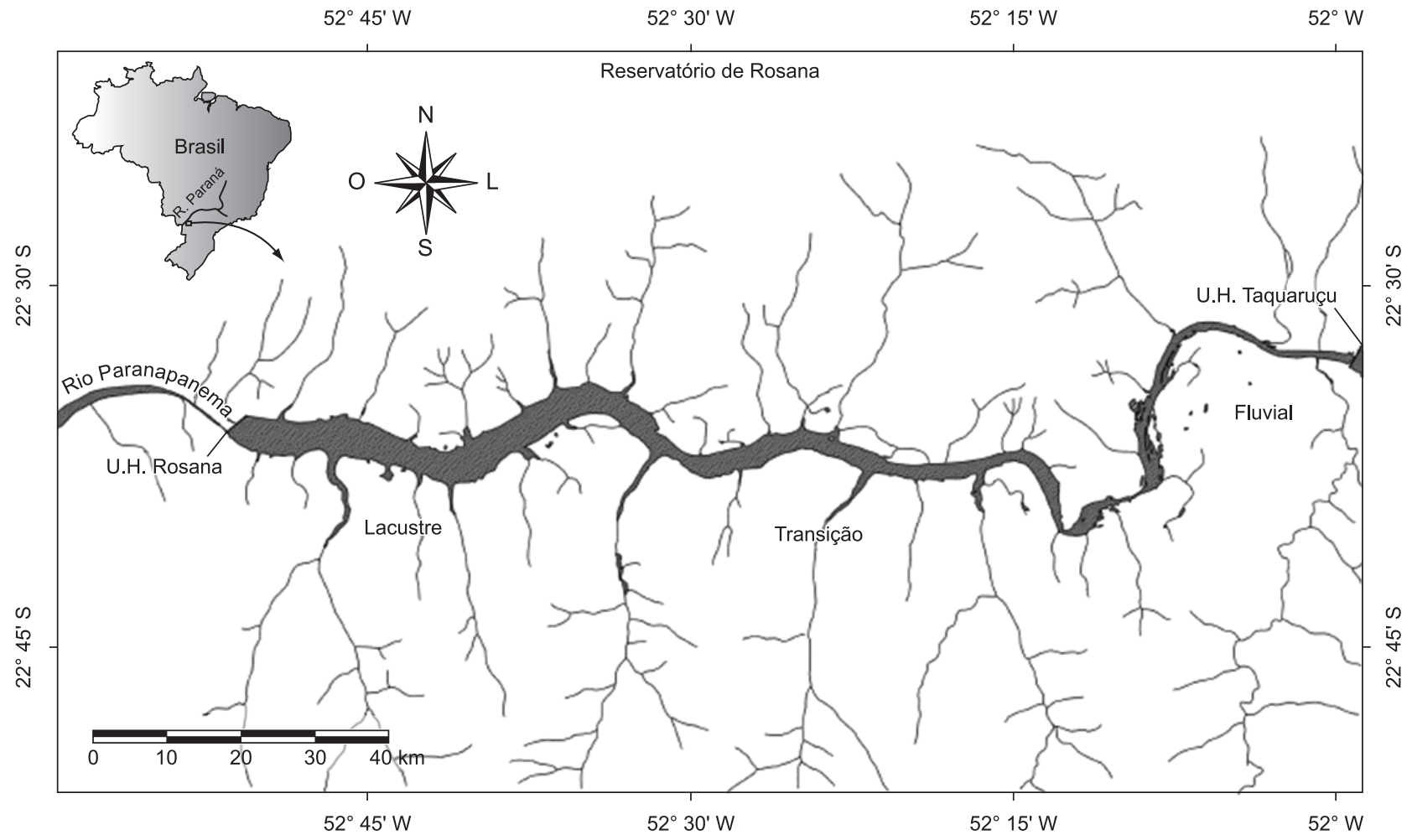

Figura 1. Localização da estação de amostragem.

Figure 1. Location of sampling station. 
do volume de água filtrada; e ii) na região litorânea - arrastos do tipo picaré, com $12 \mathrm{~m}$ de comprimento, $1,5 \mathrm{~m}$ de altura, malha de $5 \mathrm{~mm}$, e parte central dotada de um saco com malhagem $0,5 \mathrm{~mm}$, o qual foi operado em direção a margem. As amostras foram acondicionadas adequadamente e fixadas em formol $4 \%$, tamponado com carbonato de cálcio.

No laboratório, o material foi triado e as larvas foram separadas e identificadas ao menor nível taxonômico possível, segundo Nakatani et al. (2001). As densidades dos organismos capturados na região limnética foram padronizadas para um volume de $10 \mathrm{~m}^{3}$ de água filtrada (segundo Tanaka 1973, modificado por Nakatani et al. 2001), utilizando-se a seguinte equação: $\mathrm{Y}=(\mathrm{X} / \mathrm{V}) .10$, onde: $\mathrm{Y}=$ número de indivíduos por $10 \mathrm{~m}^{3} ; \mathrm{X}=$ número de indivíduos coletados; e $\mathrm{V}=$ volume de água filtrada. As densidades dos organismos capturados pelo arrasto na zona litorânea foram padronizadas para uma área de $10 \mathrm{~m}^{2}$, onde a densidade foi obtida pela razão entre o número de organismos obtidos e a área amostrada.

\section{Resultados}

Na região limnética (Tabela 1), a ordem Characiformes contribuiu com $13,5 \%$ das capturas (sendo $46 \%$ na zona fluvial, $14 \%$ na de transição e $6 \%$ na lacustre), Siluriformes com $64,7 \%$ (sendo 30,7\% na zona fluvial, $61,5 \%$ na de transição e $85 \%$ na lacustre), Perciformes com $21,5 \%$ (sendo $23 \%$ na zona fluvial, $24 \%$ na de transição e $8 \%$ na lacustre) e os Pleuronectiformes tiveram contribuição inferior a $0,5 \%$ (menos de $1 \%$ nas zonas de transição e lacustre).

Entre os Characiformes, puderam ser identificados quatro táxons ao nível específico, sendo eles: Salminus brasiliensis (Cuvier), Bryconamericus stramineus Eigenmann, Roeboides descalvadensis Fowler e Hoplias aff. malabaricus (Bloch) e dois a nível genérico, Astyanax spp. e Hyphessobrycon sp. Entre os Siluriformes, foram observadas quatro espécies: Auchenipterus osteomystax (Ribeiro), Pimelodus maculatus Lacépède, Iheringhicthys labrosus (Lütken) e Hypophthalmus edentatus (Spix). Plagioscion squamosissimus (Heckel) foi a única espécie dentre os Perciformes, assim como Catathyridium jenynsii (Günther), entre os Pleuronectiformes.

Na região litorânea (Tabela 2), a ordem Characiformes contribuiu com $90 \%$ das capturas (sendo $84 \%$ na zona fluvial, $95 \%$ na de transição e $88 \%$ na lacustre), os Siluriformes, com 5\% (sendo $12 \%$ na zona fluvial, $5 \%$ na de transição), os Gymnotiformes, com apenas $1 \%$ das capturas (contribuindo com $4 \%$ na zona fluvial) e os Perciformes somaram $4 \%$ (contribuindo com $12 \%$ na zona lacustre).

Dentro da ordem Characiformes puderam ser identificados três táxons a nível específico, $B$. stramineus, $R$. descalvadensis e Apareiodon affinis (Steindachner). Já entre os Siluriformes, foram observadas três espécies: A. osteomystax, P. maculatus e Rhamdia quelen (Quoy \& Gaimard). Entre os Perciformes, duas espécies foram identificadas: P. squamosissimus e Geophagus brasiliensis (Quoy \& Gaimard).

Tabela 1. Densidade média das larvas de peixes (larvas $/ 10 \mathrm{~m}^{3}$ ) coletadas na região limnética do reservatório de Rosana, rio Paranapanema, ao longo de um gradiente longitudinal (fluvial, transição e lacustre), nos meses de março (M), junho (J), setembro (S) e dezembro (D) de 2002. Táxons enquadrados segundo Reis et al. (2003).

Table 1. Mean density of fish larvae (larvae/10 $\mathrm{m}^{3}$ ) collected in the limnetic zone of Rosana reservoir, Paranapanema River, along a longitudinal gradient (fluvial, transition and lacustrine), in March (M), June (J), September (S) and December (D) 2002. Taxa classified according to Reis et al. (2003).

\begin{tabular}{|c|c|c|c|c|c|c|c|c|c|c|c|c|}
\hline \multirow[t]{2}{*}{ Taxa } & \multicolumn{4}{|c|}{ Fluvial } & \multicolumn{4}{|c|}{ Transição } & \multicolumn{4}{|c|}{ Lacustre } \\
\hline & $\mathbf{M}$ & $\mathbf{J}$ & $\mathbf{S}$ & D & $\mathbf{M}$ & $\mathbf{J}$ & $\mathbf{S}$ & D & $\mathbf{M}$ & $\mathbf{J}$ & $\mathbf{S}$ & D \\
\hline Characiformes $* *$ & - & - & - & - & - & - & 0,19 & - & - & - & - & 1,17 \\
\hline Anostomidae* & - & - & - & - & - & - & - & 2,78 & - & - & - & 0,29 \\
\hline \multicolumn{13}{|l|}{ Characidae } \\
\hline Astyanax spp. & - & - & - & 0,51 & - & - & - & 23,5 & - & - & - & - \\
\hline Bryconamericus stramineus & 0,82 & - & - & - & - & - & - & - & - & - & - & - \\
\hline Hyphessobrycon sp. & - & - & - & - & - & - & - & - & - & - & - & 0,29 \\
\hline Salminus brasiliensis & - & - & - & - & - & - & - & 0,31 & - & - & - & - \\
\hline Roeboides descalvadensis & - & - & - & - & - & - & - & 1,54 & - & - & - & 0,59 \\
\hline \multicolumn{13}{|l|}{ Erythrinidae } \\
\hline Hoplias aff. malabaricus & - & - & - & 0,25 & - & - & - & - & - & - & - & - \\
\hline \multicolumn{13}{|l|}{ Siluriformes } \\
\hline Heptapteridae* & - & - & - & - & - & - & - & 0,62 & - & - & - & 0,29 \\
\hline \multicolumn{13}{|l|}{ Pimelodidae } \\
\hline Hypophthalmus edentatus & - & - & - & 0,25 & - & - & - & 114 & - & - & - & 31 \\
\hline Iheringhichthys labrosus & - & - & - & - & - & - & - & 3,4 & - & - & - & 0,29 \\
\hline Pimelodus maculatus & - & - & - & 0,25 & - & - & - & 5,87 & - & - & - & - \\
\hline \multicolumn{13}{|l|}{ Auchenipteridae } \\
\hline Auchenipterus osteomystax & - & - & - & 0,51 & - & - & - & - & - & - & - & - \\
\hline \multicolumn{13}{|l|}{ Perciformes } \\
\hline \multicolumn{13}{|l|}{ Sciaenidae } \\
\hline Plagioscion squamosissimus & - & - & - & 0,77 & - & - & - & 48,8 & 0,32 & - & - & 2,64 \\
\hline \multicolumn{13}{|l|}{ Pleuronectiformes } \\
\hline \multicolumn{13}{|l|}{ Achiridae } \\
\hline Catathyridium jenynsii & - & - & - & - & - & - & - & 0,31 & - & - & - & 0,29 \\
\hline
\end{tabular}

**Espécimes identificados somente em nível de ordem; *espécimes identificados somente em nível de família.

**Specimens identified only at Order level; *specimens identified only as a Family level. 
Os táxons que apresentaram maior densidade na região limnética foram P. squamosissimus (Perciformes), H. edentatus, P. maculatus (Siluriformes), Astyanax spp. e R. descalvadensis (Characiformes), enquanto na região litorânea foram B. stramineus e A. affinis (Characiformes).

\section{Discussão}

O levantamento realizado nos diferentes ambientes do reservatório de Rosana mostrou que em termos de composição foram encontradas larvas de quinze espécies, sendo doze na região limnética e oito na litorânea. Nakatani et al. (2005) estudando o ictioplâncton de 31 reservatórios do Estado do Paraná, encontraram maior diversidade de larvas naqueles localizados no Rio Paranapanema, corroborando os resultados encontrados por Luiz et al. (2005) com peixes adultos. Segundo estes autores, além de apresentarem o maior número de espécies, estes reservatórios também estão entre os que possuem espécies com as mais diversas estratégias reprodutivas. Britto \& Carvalho (2006) consideram que ao longo deste rio, a diversidade da ictiofauna varia de 50 a 80 espécies. Na parte superior da bacia, no reservatório de Jurumirim, foram identificadas 31 espécies (Carvalho \& Silva 1999), enquanto que no baixo Paranapanema foram observadas 78 espécies no reservatório de Taquaruçu (Britto \& Carvalho 2006) e 70 em Rosana (Pelicice et al. 2005).

A distribuição transversal do ictioplâncton evidenciou na região limnética, principalmente, larvas de Siluriformes e Perciformes, influenciada pela grande abundância de $H$. edentatus (espécie que passou a ocupar as partes superiores da bacia do Paraná, após a construção do reservatório de Itaipu, que eliminou a barreira geográfica das Sete Quedas) e P. squamosissimus (espécie exótica), respectivamente. Bialetzki et al. (2005) estudaram o ictioplâncton do Rio Baía, um ambiente lêntico da bacia do alto Rio Paraná, e também verificaram elevada abundância destas espécies, que contribuíram com mais de $90 \%$ das larvas capturadas. Esta grande abundância pode ser atribuída ao tipo de desenvolvimento inicial (ovos e larvas pelágicos; Nakatani 1994) que favorece a dispersão na superfície (Holland 1986). Além disso, a alta fecundidade, as grandes populações de adultos e a baixa mortalidade larval também podem contribuir para o sucesso reprodutivo destas espécies (Araujo-Lima \& Oliveira 1998).

Na região litorânea, a predominância foi de larvas da ordem Characiformes (90\%), principalmente B. stramineus e A. affinis. Orsi et al. (2002) relatam que entre os Characiformes há um grande predomínio de espécies de pequeno porte e/ou capazes de concluir seu ciclo de vida em ambientes lênticos. A predominância de larvas de $B$. stramineus na região litorânea em todas as zonas, e também na região limnética da zona fluvial, mostra que a espécie apresenta várias estratégias, adaptando-se aos mais diversos ambientes. Esta espécie aparentemente não apresenta um padrão claro de distribuição de larvas, sendo capturada durante todo ano (Galuch et al. 2003). Entretanto, nos Rios Baía (Bialetzki et al. 2005) e Ivinheima (Reynalte-Tataje 2007), esta espécie é oportunista e sua desova ocorre na zona pelágica e suas larvas derivam rio abaixo.

As larvas de A. affinis, provavelmente estão associadas à disponibilidade de algas nesta região, tendo em vista que Santin et al. (2004) verificaram que este é o principal recurso utilizado em sua dieta. Bryconamericus stramineus e A. affinis apresentaram distribuição semelhante nas três zonas da região litorânea, evidenciando uma

Tabela 2. Densidade média das larvas de peixes (larvas $/ 10 \mathrm{~m}^{2}$ ) coletadas na região litorânea do reservatório de Rosana, Rio Paranapanema, ao longo de um gradiente longitudinal (fluvial, transição e lacustre), nos meses de março (M), junho (J), setembro (S) e dezembro (D) de 2002. Táxons enquadrados segundo Reis et al. (2003).

Table 2. Mean density of fish larvae (larvae $/ 10 \mathrm{~m}^{2}$ ) collected in the littoral region of Rosana reservoir, Paranapanema River, along a longitudinal gradient (fluvial, transition and lacustrine), in March (M), June (J), September (S) and December (D) 2002. Taxa classified according to Reis et al. (2003).

\begin{tabular}{|c|c|c|c|c|c|c|c|c|c|c|c|c|}
\hline \multirow[t]{2}{*}{ Taxa } & \multicolumn{4}{|c|}{ Fluvial } & \multicolumn{4}{|c|}{ Transição } & \multicolumn{4}{|c|}{ Lacustre } \\
\hline & $\mathbf{M}$ & $\mathbf{J}$ & $\mathbf{S}$ & D & M & $\mathbf{J}$ & $\mathbf{S}$ & D & $\mathbf{M}$ & $\mathbf{J}$ & $\mathbf{S}$ & D \\
\hline Characiformes $* *$ & 0,11 & - & - & - & - & - & - & 0,13 & - & - & - & - \\
\hline Anostomidae* & & - & & & & & & 0,13 & & & & \\
\hline \multicolumn{13}{|l|}{ Parodontidae } \\
\hline Apareiodon affinis & 0,11 & - & - & 0,13 & 0,31 & - & - & - & - & - & - & 0,38 \\
\hline \multicolumn{13}{|l|}{ Characidae } \\
\hline Bryconamericus stramineus & 0,31 & - & 0,75 & 1,28 & - & 3,76 & - & 0,68 & - & 2,37 & - & 0,5 \\
\hline Roeboides descalvadensis & - & - & - & - & - & - & - & - & - & - & - & 0,5 \\
\hline \multicolumn{13}{|l|}{ Siluriformes } \\
\hline \multicolumn{13}{|l|}{ Heptapteridae } \\
\hline Rhamdia quelen & - & - & - & - & - & - & - & 0,13 & - & - & - & - \\
\hline \multicolumn{13}{|l|}{ Pimelodidae } \\
\hline Pimelodus maculatus & - & - & - & - & - & - & - & 0,13 & - & - & - & - \\
\hline \multicolumn{13}{|l|}{ Auchenipteridae } \\
\hline Auchenipterus osteomystax & - & - & - & 0,38 & - & - & - & - & - & - & - & - \\
\hline Gymnotiformes** & - & - & - & 0,13 & - & - & - & - & - & - & - & - \\
\hline \multicolumn{13}{|l|}{ Perciformes } \\
\hline \multicolumn{13}{|l|}{ Sciaenidae } \\
\hline Plagioscion squamosissimus & - & - & - & - & - & - & - & - & - & - & - & 0,38 \\
\hline \multicolumn{13}{|l|}{ Cichlidae } \\
\hline Geophagus brasiliensis & - & - & - & - & - & - & - & - & - & - & - & 0,13 \\
\hline Danificadas & - & - & 0,5 & - & - & 0,24 & - & - & - & - & - & - \\
\hline
\end{tabular}


maior uniformidade longitudinal nesta região, no que diz respeito ao fornecimento de condições abióticas e bióticas favoráveis ao sucesso no desempenho da estratégia reprodutiva destas espécies. A captura de larvas de $P$. squamosissmus e G. brasiliensis, na região litorânea, pode ser considerada acidental, já que a primeira espécie desenvolve todo seu ciclo de vida na região limnética (Bialetzki et al. 2004) e a segunda apresenta cuidado parental (Suzuki et al. 2005).

As únicas espécies migradoras de longas distâncias para as quais foram capturadas larvas foram Salminus brasiliensis e Pimelodus maculatus (Suzuki et al., 2005), na zona fluvial da região limnética. Este resultado mostra que estas espécies devem estar utilizando áreas à montante do reservatório para a reprodução e suas larvas derivam rio abaixo, até encontrarem ambientes adequados ao seu desenvolvimento. Baumgartner et al. (2008) também encontram larvas desta espécie em tributários acima do reservatório de Itaipu. De acordo com Agostinho \& Zalewski (1996), a manutenção da ictiofauna na planície de inundação do alto Rio Paraná, especialmente das espécies tipicamente migradoras, só será possível em reservatórios com trechos de relativas distâncias livres ou tributários apropriados para este fim.

Analisando a distribuição longitudinal e transversal do ictioplâncton no reservatório de Rosana, fica evidente que a estrutura das assembleias de larvas de peixes é influenciada por vários fatores intrínsecos e extrínsecos. Assim, há de se considerar a importância dos diferentes ambientes analisados, pois estes podem ser utilizados pelas diferentes espécies para completar seu ciclo de vida, dependendo ou não da fase de desenvolvimento nas quais elas se encontram.

\section{Agradecimentos}

Os autores agradecem ao Núcleo de Pesquisas em Limnologia, Ictiologia e Aquicultura (Nupélia/UEM) pelo apoio logístico; aos funcionários da UEM: João Dirço Latini, Valmir Alves Teixeira, Valdir Aparecido Capatti, Francisco Alves Teixeira, José Ricardo Gonçalves e Valdecir Rodolfo Casari pela coleta do material em campo; ao desenhista Jaime Luiz Lopes Pereira pela confecção do mapa; aos amigos do Laboratório de Ictioplâncton/Nupélia/UEM, pelo auxílio na análise dos dados. Ao projeto financiado pelo Programa PRONEX/ $\mathrm{CNPq}$ (Produtividade em reservatórios: relação com o estado trófico e a predação - UEM/Processo no 0888/98).

\section{Referências Bibliográficas}

AGOSTINHO, A.A. \& ZALEWSKI, M.A. 1996. Planície alagável do alto rio Paraná: importância e preservação. EDUEM, Maringá, 100p.

ARAÚJO-LIMA, C.A.R.M. \& OLIVEIRA, E.C. 1998. Transport of larval fish in the Amazonian. J. Fish Biol. 53:297-306.

BAUMGARTNER, G., NAKATANI, K., GOMES, L.C., BIALETZKI, A., SANCHES, P.V. \& MAKRAKIS, M.C. 2008. Fish larvae from the upper Paraná River: do abiotic factors affect larval density? Neot. Ichthyol. 6:551-558

BIALETZKI, A., NAKATANI, K., SANCHES, P.V. \& BAUMGARTNER, G. 2004. Eggs and larvae of the 'curvina' Plagioscion squamosissimus (Heckel, 1840) (Osteichthyes, Sciaenidae) in the Baía River, Mato Grosso do Sul State, Brazil. J. Plankton Res. 26:1327-1336.

BIALETZKI, A., NAKATANI, K., SANCHES, P.V., BAUMGARTNER, G. \& GOMES, L.C. 2005. Larval fish assemblage in the Baía River (Mato Grosso do Sul State, Brazil): temporal and spatial patterns. Env. Biol. Fish. 73:37-47.

BRITTO, S.G.C. \& CARVALHO, E.D. 2006. Ecological attributes of fish fauna the Taquaruçu reservoir, Paranapanema River (Upper Paraná, Brazil): composition and spatial distribution. Acta Limnol. Bras. 18:377388.

CARVALHO, E.D. \& SILVA, V.F.B. 1999. Aspectos ecológicos da ictiofauna e da produção pesqueira do reservatório de Jurumirim (Alto do rio Paranapanema, São Paulo). In Ecologia de Reservatórios: estrutura, função e aspectos sociais (R. Henry ed.). FUNDIBIO; FAPESP, Botucatu, p.769-800.
ESTEVES, F.A. 1998. Fundamentos de limnologia. Interciência-FINEP, Rio de Janeiro, 602p.

GALUCH, A.V., SUIBERTO, M.R., NAKATANI, K., BIALETZKI, A. \& BAUMGARTNER, G. 2003. Desenvolvimento inicial e distribuição temporal de larvas e juvenis de Bryconamericus stramineus Eigenmann, 1908 (Osteichthyes, Characidae) na planície alagável do alto rio Paraná, Brasil. Acta Sci. 25:335-343.

HOLLAND, L.E. 1986. Distribution of early life history of fishes in selected pools of the Upper Mississipi River. Hydrobiol. 136:121-130.

JÚLIO JÚNIOR, H.F., THOMAZ, S.M., AGOSTINHO, A.A. \& LATINI, J.D. 2005. Distribuição e caracterização dos reservatórios. In Biocenoses em Reservatórios (Padrões espaciais e temporais) (L. Rodrigues, S.M Thomaz, A.A. Agostinho \& L.C. Gomes, eds.). RiMa, São Carlos, p.1-16.

LUIZ, E.A., PETRY, A.C., PAVANELLI, C.S., JULIO-Jr, H.F., LATINI, J.D. \& DOMINGUES, V.M. 2005. As assembléias de peixes de reservatórios do Estado do Paraná e bacias limítrofes. In Biocenoses em Reservatórios (Padrões espaciais e temporais) (L. Rodrigues, S.M. Thomaz, A.A. Agostinho \& L.C. Gomes, eds.). RiMa, São Carlos, p.169-184.

MILLER, T. 2002. Assemblages, communities, and species interactions. In Fishery Science. The unique contributions of early life stages (L.E. Fuimann \& R.G. Werner, eds.). Blackwell Publishing, Oxford, p. 183-205.

NAKATANI, K. 1994. Estudo do ictioplâncton no reservatório de Itaipu (rio Paraná-Brasil): levantamento das áreas de desova. Tese de Doutorado, Universidade Federal do Paraná, Curitiba, Paraná.

NAKATANI, K., AGOSTINHO, A.A., BAUMGARTNER, G., BIALETZKI, A., SANCHES, P.V., MAKRAKIS, M.C. \& PAVANELLI, C.S. 2001. Ovos e larvas de peixes de água doce: desenvolvimento e manual de identificação. EDUEM, Maringá, 378p.

NAKATANI, K., BIALETZKI, A., SANTIN, M., BORGES, R.Z., ASSAKAWA, L.F., ZIOBER, S.R., KIPPER, D., GALUCH, A.V. \& SUIBERTO, M.R. 2005. Ocorrência e abundância de larvas e juvenis de peixes em reservatórios. In Biocenoses em reservatórios (padrões espaciais e temporais) (L. Rodrigues, S.M. Thomaz, A.A. Agostinho \& L.C. Gomes, eds.). RiMa, São Carlos, p.253-268.

OLIVEIRA, E.F. \& GOULART, E. 2000. Distribuição espacial de peixes em ambientes lênticos: interação de fatores. Acta Sci. 22:445-453.

ORSI, M.L., SHIBATTA, O.A. \& SILVA-SOUZA, A.T. 2002. Caracterização biológica de populações de peixes do rio Tibagi, localidade de Sertanópolis. In A Bacia do Rio Tibagi (M.E. Medri, O.A. Shibatta, E. Bianchini \& J.A. Pimenta, eds.). Edição dos editores, Londrina, p.425-432.

PELICICE, F.M., AGOSTINHO, A.A. \& THOMAZ, S.M. 2005. Fish assemblages associated with Egeria in a tropical reservoir: investigating the effects of plant biomass and diel period. Acta Oecol. 27:9-16.

REIS, R.E., KULLANDER, S.O. \& FERRAIS-Jr., C.J. 2003. Check list of the freshwater fishes of South and Central America. EDIPUCRS, Porto Alegre, 729p.

REYNALTE-TATAJE, D.A. 2007. Influência inter e intra anual de variáveis ambientais sobre a estrutura da comunidade ictioplanctônica em duas bacias hidrográficas brasileiras. Tese de doutorado em Ecologia de Ambientes Aquáticos Continentais, Universidade Estadual de Maringá, Paraná, Brasil.

SANTIN, M., BIALETZKI, A. \& NAKATANI, K. 2004. Mudanças ontogênicas no trato digestório e dieta de Apareiodon affinis (Steindachner, 1879) (Osteichthyes, Parodontidae). Acta Sci. 26:291-298.

STRAŠKRABA, M., TUNDISI, J.G. \& DUNCAN, A. 1993. State of the art of reservoir limnology and water quality management. In Comparative reservoir limnology and water quality management (M. Straškraba, J.G. Tundisi \& A. Duncan, eds.). Kluwer Academic Publishers, Dordrecht, p.213-288.

SUZUKI, H.I., BULLA, C.K. \& AGOSTINHO, A.A. 2005. Estratégias reprodutivas de assembléias de peixes em reservatórios. In Biocenoses em Reservatórios (Padrões espaciais e temporais) (L. Rodrigues, S.M. Thomaz, A.A. Agostinho \& L.C. Gomes, eds.). RiMa, São Carlos, p.223-236. 
Kipper, D. et al.

TANAKA, S. 1973. Stock assessment by means of ichthyoplankton surveys. FAO Fish. 122:33-51.

THORNTON, K.W., KIMMEL, B.L. \& PAINE, F.E. 1990. Reservoir limnology: ecological perspectives. John Wiley \& Sons, New Jersey, 246p.
TUNDISI, J.G. 1999. Reservatórios como sistemas complexos: teoria, aplicações e perspectivas para usos múltiplos. In Ecologia de Reservatórios: estrutura, função e aspectos sociais (R. Henry, ed.). FUNDIBIO, São Paulo; Botucatu, p. 19-38.

Recebido em 05/08/2010 Versão reformulada recebida em 08/12/2010

Publicado em 10/01/2011 